\title{
CORRELATION BETWEEN PERIODONTAL DISEASE INDICES AND LUNG CANCER IN GREEK ADULTS: A CASE - CONTROL STUDY
}

\author{
N.A. Chrysanthakopoulos \\ Maxillofacial and Oral Surgery, 401 General Military Hospital of Athens, Athens 11527, Greece \\ Department of Pathological Anatomy, Medical School, University of Athens, Athens 11528, Greece
}

\begin{abstract}
Aim: The aim of the present case - control study was to examine the possible associations between periodontal disease indices and the risk of lung cancer development in a sample of Greek out-patients referred to a medical and a dental private practice. Materials and Methods: A total of 200 individuals were interviewed and underwent an oral clinical examination, and 64 of them were suffered from several histological types of lung cancer. The estimation of the possible associations between lung cancer as a dependent variable and periodontal disease indices as independent ones was carried out by using a multiple regression analysis model. Results: Probing pocket depth (odds ratio $(O R)=2.72,95 \%$ confidence interval $(C I) 1.05-7.06)$, clinical attachment loss $(O R=$ 3.51, 95\% CI 1.30-9.47) bleeding on probing $(\mathrm{OR}=1.93,95 \%$ CI $0.98-3.81)$ were significantly associated with the risk of developing lung cancer. Smoking $(\mathrm{OR}=\mathbf{2 . 4 9}, 95 \%$ CI 1.20-5.17) was significantly associated with the mentioned risk, whereas it was consisted as a confounder regarding the estimated associations between moderate/severe clinical attachment loss and presence of bleeding on probing with the risk of developing lung cancer. Conclusion: Probing pocket depth as an index for periodontal disease severity was statistically significantly associated with the risk of developing lung cancer.
\end{abstract}

Key Words: periodontal disease, lung cancer, adults, chronic inflammation, risk factor.

Lung cancer (LC) is one of the most deadly of all types of cancer, and the $5^{\text {th }}$ cause of mortality nowadays in industrialized countries and occurs as a result of genetic, environmental and behavioral risk factors [1, 2]. Common genetic factors are age, male gender, genetic predisposition, enzymes polymorphism and other unknown factors. The environmental and behavioral risk factors include smoking, exhibition in inhaled gases/ polluted air and previous pulmonary diseases such as chronic obstructive pulmonary disease, tuberculosis (TBC) and lung fibrosis.

The last suggestion led to the hypothesis that systemic chronic inflammation could play an essential role in development and/or disease progression. Chronic inflammation may enhance mutagenesis and cell proliferation, inhibit apoptosis, reduce the adaptation to oxidative stress, promote angiogenesis, and increase the secretion of inflammatory mediators [2].

Periodontal disease (PD) and especially periodontitis is a common and destructive disease of the oral cavity, leads to tooth loss, its development is a complex process that occurs over a long period of time, as a result of bacterial infection and inflammation which spreads in periodontal fibers and alveolar bone, the supporting structures of teeth [3]. Periodontal tissues infection leads to systemic effects and an increase in circulating levels of inflammatory biomarkers, that correlate directly with the severity of disease, such as C-reactive protein, interleukin-1 and -6 . The

Submitted: January 18, 2016.

Correspondence: Fax: 0030-2610-225288;

E-mail: nikolaos_c@hotmail.com; nchrysant@med.uoa.gr Abbreviations used: AC - adenocarcinoma; BOP - bleeding on probing; CAL - clinical attachment loss; LC - lung cancer; LCC large cell carcinoma; PD - periodontal disease; PPD - probing pocket depth; SCC - squamous cell carcinoma; SCLC - small cell lung carcinoma; TBC - tuberculosis. biological mechanisms of those associations remain unknown although various inflammatory biomarkers, such as the mentioned may be involved as mediators of systemic inflammation $[4,5]$. To be more specific, it remains unknown whether systemic inflammation, invasion of pathogenic bacteria in systemic circulation or immune response to periodontal infection may affect the risk for various systemic diseases development, including cancer [4].

Recent epidemiologic reports has linked periodontal pathogens to several systemic diseases, such as cardiovascular disease, diabetes mellitus, respiratory disease, and systemic infections. Those associations possibly mediated through biomarkers of systemic infection and inflammation [6, 7].

A possible correlation between PD and cancer risk in different locations, most notably in the oral cavity, upper gastrointestinal system, lung, pancreas, and other organs has also been proposed [8-14]. In two of those prospective studies $[9,10]$ a correlation between tooth loss, as another PD indicator, and the risk of cancer was recorded, however such correlations regarding LC remain unclear [10]. Tooth loss is caused by dental caries and PD, however the distribution of all causes depends on age and other variables. Chronic periodontitis is responsible for tooth loss in older individuals, whereas in younger ages the main cause is dental caries. Therefore, although tooth loss could be an indicator of PD, its correlation is not always strong [15].

Poor oral health or hygiene, as indicators of PD, are possible risk factors for cancer in different organs according to recent epidemiological studies $[8,9,16$, 17]. It has been hypothesized that $P D$ increases the risk of LC through bacterial load and subsequent chronic systemic inflammation $[9,10]$. However to our knowledge previous studies have not objectively evaluated the role of PD in LC or precancerous lesions. Several reasons 
could explain a possible link between periodontitis and cancer. Both diseases have several risk factors in common, while smoking, advanced age and low socioeconomic status have been implicated in cancer and periodontitis [18].

The present retrospective case - control study was carried out to examine the possible correlation between PD indices such as probing pocket depth (PPD), clinical attachment loss (CAL) and bleeding on probing (BOP) and the LC risk in a sample of Greek adults.

\section{MATERIALS AND METHODS}

Study sample. The material consisted of 200 subjects, 126 males and 74 females. Cases and controls were selected from a private medical and dental practice, completed a health questionnaire and underwent an oral clinical examination.

Patients selection criteria. Patients and controls was necessary to have a mean of 20 natural teeth, since large numbers of missing teeth could lead to overor underestimate the dental variables and the possible associations that were under consideration and the criteria of established periodontitis [19], which referred to at least 2 teeth with $\mathrm{CAL} \geqslant 6 \mathrm{~mm}$ and more that one site with PPD $\geqslant 5 \mathrm{~mm}$.

None of the participants had received scaling and root planning procedures or periodontal treatment during the previous 6 months or prescription of anti-inflammatory or systemic antibiotics or other systemic drugs the previous 6 weeks [20]. In order to avoid as much as possible, potential confounding influences on the study parameters, individuals with acute infections, cardiovascular diseases, diabetes mellitus, rheumatoid arthritis, immuno-suppressed patients because of haematological malignancy or recent transplantation and those who received treatment for the mentioned diseases, liver cirrhosis and concurrent medication with general glucocorticoids were excluded from the study. They also excluded patients with advanced LC under medical treatment, patients with lung metastases of a primary focus at a different location, patients diagnosed with mesothelioma or other focuses in the region of head-neck-thorax (carcinogenesis field theory [21]). These criteria were applied because of potential effects on the oral tissues. Hospital patients did not include or patients with several location of cancer in which smoking is considered as a proven risk factor such as larynx cancer, nasopharyngeal cancer, etc.

The patients' group was consisted of individuals in which the diagnosis of LC was set initially by histological examination during the endoscopic procedure and they had been given instructions regarding their oral hygiene after diagnosis and before the application of any treatment method, such as surgery, radiotherapy or chemotherapy.

Controls group selection was carried out by the friendly and collegial environment of cases group in an effort to control potential confounders such as socio-economic level.
Oral clinical examination. One well trained and calibrated dentist performed the examinations at the mentioned private practices. The clinical measurements concerned the following variables: For each tooth, except for the $3^{\text {rd }}$ molars and the remaining roots PPD, CAL and BOP were measured by a William's 12 PCP probe (PCP 10-SE, Hu-Friedy Mfg. Co. Inc., Chicago, IL, USA) at six sites (facial, lingual, distofacial, mesio-facial, disto-lingual and mesio-lingual).

The presence of PPD was classified as follows [22]: score 0: moderate periodontal pockets, 4-6.0 mm, and score 1: advanced periodontal pockets, $>6.0 \mathrm{~mm}$.

The severity of CAL classified as follows [23]: score 0: mild,1-2.0 mm of attachment loss, and score 1: moderate/severe, $\geqslant 3.0 \mathrm{~mm}$ of attachment loss. The record for PPD and CAL measurements concerned the immediate full millimeter.

The presence/absence of BOP was classified as follows: score 0: absence of BOP, and score 1: presence of BOP and deemed positive if it occurred within 15 s of probing.

Questionnaire. All participants were filled in a selfadministered questionnaire that included variables such as age, gender, smoking status (active, former/ no-smokers), socio-economic and educational level and data regarding their general medical history with reference to medication, several chronic systemic disorders and the dental follow-up frequency.

A randomly chosen sample of 40 (20\%) individuals was re-examined clinically by the same dentist after 3 weeks in order to establish the intra-examiner variance. After consideration of the code numbers of the double examined individuals no differences were recorded between the $1^{\text {st }}$ and the $2^{\text {nd }}$ clinical assessment (Cohen's Kappa = 0.98) whereas for the mentioned time period no oral hygiene instructions were given to the participants.

Ethical consideration. The present study was not an experimental one. In Greece only experimental studies must be reviewed and approved by authorized committees (Dental Schools, Greek Dental Associations, Ministry of Health, etc). Individuals who agreed to participate in the present study signed an informed consent form.

Statistical analysis. For each individual, case and control the worst values of PPD and CAL at the six sites per tooth and the presence/absence of BOP were recorded and coded as dichotomous variables. Current and former smokers were coded as 1, individuals with a high socio-economic (income/monthly $\geqslant 1000 €$ ) and educational (graduated from University/College) level were coded as 0 , males participants were coded as 1 , individuals that reported genetic predisposition for LC, history of previous chronic pulmonary disease and a regular dental follow-up were coded as 1. Age groups distribution was coded as 0, 1, 2 and 3 for ages 48-49, 50-59, 60-69 and 70+, respectively.

Univariate analysis was carried out to test the relationship between the independent variables examined and the LC risk, separately, by using $x^{2}$ test. Multivariate regression analysis was carried out to model the associations between the dependent variable, LC, and 
independent ones that were determined by the enter method. Adjusted odds ratios (OR's) and 95\% confidence interval $(\mathrm{Cl})$ were also calculated. Finally, the independent variables were included to stepwise method in order to estimate gradually the variables that showed significant correlations with the dependent one. The statistical method Cohran's and Mantel - Haenszel's was applied, in an effort to control possible con-founders, in order to avoid biased secondary associations. Statistical analysis was performed using the statistical package of SPSS ver.17.0. A p value less than $5 \%(p<0.05)$ was considered to be statistically significant.

\section{RESULTS}

The mean age of the sample was $61.4 \pm 4.2$ years.

The most frequent histological type in males was squamous cell carcinoma (SCC) $(47.8 \%)$, followed by small cell lung carcinoma (SCLC) $(30.4 \%)$, adenocarcinoma (AC) $(13.0 \%)$ and large cell carcinoma (LCC) $(8.8 \%)$, whereas in females AC (44.4\%) followed by SCC (33.3\%), SCLC (16.7\%) and LCC (5.6\%).

Table 1 presents univariate analysis of cases and controls regarding the examined variables. PPD, BOP, history of previous chronic pulmonary disease, smoking and irregular dental follow-up were statistically significantly associated with LC risk. Table 1 also presents unadjusted OR's and 95\% Cl.

Table 1. Univariate analysis of cases and controls regarding each independent variable examined

\begin{tabular}{|c|c|c|c|c|c|}
\hline Variables & $\begin{array}{c}\text { Cases, } \\
\mathrm{n}(\%)\end{array}$ & $\begin{array}{c}\text { Controls, } \\
n(\%)\end{array}$ & $\begin{array}{c}\mathrm{p} \\
\text { value }\end{array}$ & OR & $95 \% \mathrm{Cl}$ \\
\hline \multicolumn{6}{|l|}{ Gender: } \\
\hline Males & $46(71.9)$ & $80(58.8)$ & 0.075 & 1.79 & $0.94-3.40$ \\
\hline $\begin{array}{l}\text { Females } \\
\text { Age (years): }\end{array}$ & $18(28.1)$ & $56(41.2)$ & & & \\
\hline $45-49$ & $4(6.3)$ & $10(7.4)$ & 0.578 & - & - \\
\hline $50-59$ & $11(17.2)$ & $32(23.5)$ & & & \\
\hline $60-69$ & $41(64.0)$ & $73(53.7)$ & & & \\
\hline $\begin{array}{l}70+ \\
\text { Socio-economic level: }\end{array}$ & $8(12.5)$ & $21(15.4)$ & & & \\
\hline Low & $38(59.4)$ & $91(66.9)$ & 0.300 & 1.38 & $0.75-2.56$ \\
\hline $\begin{array}{l}\text { High } \\
\text { Educational level: }\end{array}$ & $26(40.6)$ & $45(33.1)$ & & & \\
\hline Low & $52(81.3)$ & 97 (71.3) & 0.133 & 0.57 & $0.28-1.19$ \\
\hline $\begin{array}{l}\text { High } \\
\text { Smoking: }\end{array}$ & $12(18.7$ & $39(28.7)$ & & & \\
\hline No & $14(21.9)$ & $59(43.4)$ & $0.003^{*}$ & 2.74 & $1.38-5.42$ \\
\hline Yes & $50(78.1)$ & $77(56.6)$ & & & \\
\hline Cancer family history: & & & & & \\
\hline No & $50(78.1)$ & $117(86.0)$ & & & \\
\hline Yes & $14(21.9)$ & $19(14.0)$ & 0.160 & 1.72 & $0.80-3.71$ \\
\hline $\begin{array}{l}\text { History of previous pul- } \\
\text { monary disease: }\end{array}$ & & & & & \\
\hline No & $33(51.6)$ & $99(72.8)$ & $0.003^{*}$ & 2.51 & $1.35-4.67$ \\
\hline Yes & $31(48.4)$ & $37(27.2)$ & & & \\
\hline Annual dental follow-up: & & & & & \\
\hline$<2$ times or no/year & $27(42.2)$ & $38(27.9)$ & & & \\
\hline $\begin{array}{l}2 \text { times/year } \\
\text { Periodontal pockets: }\end{array}$ & $37(57.8)$ & $98(72.1)$ & $0.045^{\star}$ & 0.53 & $0.29-0.99$ \\
\hline Depth 4.0-6.0 mm & $46(71.9)$ & $116(85.3)$ & & & \\
\hline $\begin{array}{l}\text { Depth > } 6.0 \mathrm{~mm} \\
\text { CAL: }\end{array}$ & $18(28.1)$ & $20(14.7)$ & $0.024^{\star}$ & 2.27 & $1.10-4.68$ \\
\hline Mild $1-2.0 \mathrm{~mm}$ & $46(71.9)$ & $107(78.7)$ & & & \\
\hline Moderate/severe & & & 0.290 & 1.44 & $0.73-2.86$ \\
\hline $\begin{array}{l}\geqslant 3.0 \mathrm{~mm} \\
\text { BOP: }\end{array}$ & $18(28.1)$ & $29(21.3)$ & & & \\
\hline No & $22(47.8)$ & $74(54.4)$ & $0.008^{\star}$ & 2.28 & $1.23-4.22$ \\
\hline Yes & $42(52.2)$ & $62(45.6)$ & & & \\
\hline
\end{tabular}

Note: *p value: statistically significant.
After performance of the first method (step 1a) of the regression model it was found that all the examined variables except for dental follow-up and age were significantly associated with LC risk, according to the OR's. Statistically significantly associations were recorded between moderate/severe CAL, BOP and risk of developing LC (Table 2). Table 2 also presents adjusted OR's with $95 \% \mathrm{Cl}$. The final method (stepwise/step 7a) showed that smoking, PPD, CAL and BOP were significantly associated with LC risk. PPD was also significantly associated with LC risk after adjusting for confounders, such as smoking and dental follow-up (Table 3).

Table 2. Presentation of correlation between independent variables and LC according to Enter (first step) and Wald method (backward) of multiple logistic regression analysis model

\begin{tabular}{|c|c|c|c|c|c|c|c|c|c|}
\hline \multirow[t]{2}{*}{ Step } & \multirow[t]{2}{*}{ Variables } & \multirow[t]{2}{*}{ B } & \multirow[t]{2}{*}{ S.E. } & \multirow[t]{2}{*}{ Wald } & \multirow[t]{2}{*}{$\mathrm{df}$} & \multirow[t]{2}{*}{ Sig. } & \multirow{2}{*}{$\begin{array}{l}\text { Exp } \\
\text { (B) }\end{array}$} & \multicolumn{2}{|c|}{$\begin{array}{c}95 \% \mathrm{Cl} \text { for } \\
\operatorname{EXP}(\mathrm{B})\end{array}$} \\
\hline & & & & & & & & Lower & Upper \\
\hline \multirow[t]{16}{*}{$1 a$} & Gender & 0.548 & 0.373 & 2.158 & 1 & 0.142 & 1.730 & 0.833 & 3.596 \\
\hline & Smoking & 0.618 & 0.416 & 2.201 & 1 & 0.138 & 1.854 & 0.820 & 4.193 \\
\hline & Socio-eco- & & & & & & & & \\
\hline & nomic level & 0.448 & 0.400 & 1.256 & 1 & 0.262 & 1.565 & 0.715 & 3.427 \\
\hline & level & -0.238 & 0.486 & 0.240 & 1 & 0.624 & 0.788 & 0.304 & 2.041 \\
\hline & Age & 0.031 & 0.025 & 1.540 & 1 & 0.215 & 1.032 & 0.982 & 1.084 \\
\hline & Cancer family & & & & & & & & \\
\hline & history & 0.011 & 0.477 & 0.001 & 1 & 0.981 & 1.011 & 0.397 & 2.576 \\
\hline & $\begin{array}{l}\text { History of pre- } \\
\text { vious pulmo- }\end{array}$ & & & & & & & & \\
\hline & $\begin{array}{l}\text { nary disease } \\
\text { Annual dental }\end{array}$ & 0.558 & 0.384 & 2.109 & 1 & 0.146 & 1.747 & 0.823 & 3.711 \\
\hline & follow-up & -0.688 & 0.369 & 3.474 & 1 & 0.062 & 0.502 & 0.244 & 1.036 \\
\hline & BOP & 0.760 & 0.366 & 4.310 & 1 & 0.038 & 2.139 & 1.043 & 4.385 \\
\hline & Periodontal & & & & & & & & \\
\hline & pockets & 0.817 & 0.510 & 2.563 & 1 & 0.109 & 2.264 & 0.833 & 6.156 \\
\hline & $\mathrm{CAL}$ & 1.162 & 0.524 & 4.912 & 1 & 0.027 & 3.197 & 1.144 & 8.937 \\
\hline & Constant & 5.364 & 1.754 & 9.358 & 1 & 0.002 & 0.005 & & \\
\hline \multirow[t]{7}{*}{$7 a$} & Smoking & 0.913 & 0.373 & 6.008 & 1 & 0.014 & 2.492 & 1.201 & 5.173 \\
\hline & $\begin{array}{l}\text { Annual dental } \\
\text { follow-un }\end{array}$ & -0.588 & 0351 & 2803 & 1 & 0.094 & 0555 & 0279 & 1106 \\
\hline & BOP & 0.656 & 0.347 & 3.575 & 1 & 0.059 & 1.927 & 0.976 & 3.805 \\
\hline & Periodontal & & & & & & & & \\
\hline & pockets & 1.002 & 0.486 & 4.259 & 1 & 0.039 & 2.724 & 1.052 & 7.056 \\
\hline & $\mathrm{CAL}$ & 1.256 & 0.506 & 6.163 & 1 & 0.013 & 3.513 & 1.303 & 9.474 \\
\hline & Constant & 3.134 & 0.653 & 23.001 & 1 & 0.000 & 0.044 & & \\
\hline
\end{tabular}

Table 3. Application of Cohran's and Mantel - Haenszel's, statistical method for controlling possible confounders

\begin{tabular}{lcc}
\hline \multicolumn{1}{c}{ Variables } & Exp (B) & $95 \% \mathrm{Cl}$ \\
\hline Periodontal pockets & & \\
Non-smokers & 2.279 & $0.574-9.047$ \\
Smokers & 6.708 & $2.182-20.622$ \\
CAL & & \\
Non-smokers & 2.138 & $0.652-7.006$ \\
Smokers & 2.205 & $1.051-4.627$ \\
BOP & & \\
Non-smokers & 2.514 & $0.778-8.121$ \\
Smokers & 2.416 & $1.143-5.106$ \\
Periodontal pockets & & \\
Regular dental follow-up annually & 2.745 & $1.043-7.227$ \\
Irregular dental follow-up annually & 21.048 & $2.585-171.392$ \\
CAL & & \\
Regular dental follow-up annually & 3.639 & $1.298-10.205$ \\
Irregular dental follow-up annually & 13.520 & $1.644-111.154$ \\
BOP & & \\
Regular dental follow-up annually & 2.041 & $0.921-4.520$ \\
Irregular dental follow-up annually & 4.000 & $1.413-11.327$ \\
\hline
\end{tabular}

\section{DISCUSSION}

The present case - control research showed that deep periodontal pockets were associated with an increased LC risk, after controlling for possible 
confounders such as smoking and dental follow-up. Despite the fact that more investigation is required in order to confirm such findings, the current observations suggest that improvement of oral hygiene and smoking cessation could be an effective preventive measure against LC development.

The possible influence of gender as a cancer risk factor is known, however it is considered as a confounder. The results showed no association between gender and LC risk, finding that was in accordance with those from previous reports $[9,10]$.

Similarly, age is also considered as a confounder, although older individuals are in a higher risk for total cancer, LC [24, 25], initiation and progression of PD [26]. No association was found between age and $\mathrm{LC}$ risk in the present study.

Another crucial confounder is socio-economic level, however, it has not been proven its possible role as a LC risk factor. Its role is indirect in cases of previous pulmonary diseases such as TBC that is associated with a lower socio-economic level and LC development [27, 28]. No association was observed in the current study between those variables examined.

The possible role of educational level as a risk factor of developing LC has not been investigated according to previous reports. However, it is supposed that high-educated individuals take care of their own oral hygiene more than low-educated ones [29, 30]. No association was recorded between educational level and LC risk in the current study. There is strong evidence suggesting a genetic predisposition for LC. Studies of familial aggregation have shown familial risk on the same order of that reported for breast and colon cancer [31, 32]. The results of the present study did not confirm such an association.

Smoking is considered as a causal risk factor of total cancer and LC [33]. It has been shown that smoking is associated with SCC and SCLC, mainly, and in less cases with lung AC. It is still remaining unknown the reasons why only $15 \%$ of smokers develop LC [34]. The current study confirmed its role as a causal risk factor. On the other hand smoking is considered as a risk factor for PD development and progression [35, 36] and a proven confounder as well. Based on the mentioned suggestions the statistical method of adjustment Cohran's and Mantel - Haenszel's was carried out to assess if possible significant correlations between both diseases could be attributed to smoking status or not. It was found that smoking was a confounder of CAL and BOP.

According to the results PPD was associated with an increased LC risk after controlling for certain confounders such as smoking and socio-economic status. Similar reports that have investigated the possible associations between PD indices and LC risk, or total cancer have not been carried out, whereas the majority of the available studies are prospective and have based on questionnaires and self-reported data.

Arora et al. [37] were found that individuals with PD showed an increased LC risk however after con- trolling for certain confounders such as gender, age, socio-economic and educational status the mentioned association was not found to be statistically significant. That research was based on a questionnaire and selfreported information regarding periodontal tissues condition examined and different PD indices were used.

In another prospective study among health professionals in which a self-reported questionnaire was used for estimation of periodontal tissues status [9], PD was significantly associated with LC risk after controlling for smoking and several risk factors. The main finding was that a limited number of remaining teeth ( $0-16$ vs 32) was associated with an increased LC risk.

Hujoel et al. [8] based on Russel Index for PD definition were found that individuals with PD had an increased risk of total cancer and a significantly increased LC risk, finding that was not confirmed in never smokers.

A similar research in Japan [38] reported that a small number of remaining teeth was associated with an increased LC risk.

An important factor that may be taken into account during the design process of such studies, is the epidemiological phenomenon of "confounding". Both diseases, PD and cancer share some common risk factors such as smoking and socio-economic status. Consequently, a correlation between both diseases would be expected even if a causal link did not exist. Confounding may also occur through unknown factors, for example a genetic predisposition, or mutual risk factors. However, the question still remains whether the association between PD and cancer is causal or is con-founded by unmeasured factors.

Another practical problem is the accuracy definition of PD which is essential to establish on reliable and reproductive indices [39]. Data of such studies have carried out based on prospective or retrospective methodology in an attempt to control possible systematic biases, selection biases mainly, and confounding. However, smoking remains a possible interpretation as the correlations that have reported concerned smokers, whereas no correlations have been reported between PD and cancer in non-smokers. It is important to highlight that the decision on including older individuals who have at least 20 remaining natural teeth, may lead to an under-estimation of older individuals with previous PD and who may have had teeth extracted for periodontal reasons. In addition, it is essential to be noted that there was not any chance of benchmarking between the findings of the current study with those of similar previous studies, whereas on the other hand the present study was a first attempt to approach that possible correlation in Greece.

In conclusion, PD parameters such as deep periodontal pockets were associated with an increased risk of developing LC.

The present study constitutes a part of my MSc Thesis which was announced on May 2015 at Medical School, University of Athens. 


\section{REFERENCES}

1. Causes of death mortality and global health estimates. WHO report, 2012.

2. Davila DG, Williams DE. The etiology of lung cancer. Mayo Clin Proc 1993; 68: 170-82.

3. Papapanou PN. Periodontal diseases: epidemiology. Ann Periodontol 1996; 1: 1-36.

4. Renvert S, Lindahl C, Roos-Jansåker AM, et al. Shortterm effects of an antiinflammatory treatment on clinical parameters and serum levels of c-reactive protein and proinflammatory cytokines in subjects with periodontitis. J Periodontol 2009; 80: 892-900.

5. Vidal F, Figueredo CM, Cordovil I, et al. Periodontal therapy reduces plasma levels of interleukin-6, C-reactive protein, and fibrinogen in patient a with severe periodontitis and refractory arterial hypertension. J Periodontol 2009; 80: 786-91.

6. Holmstrup P, Poulsen AH, Andersen L, et al. Oral infections and systemic diseases. Dent Clin North Am 2003; 47: 575-98.

7. Teng YT, Taylor GW, Scannapieco F, et al. Periodontal health and systemic disorders. J Can Dent Assoc 2002; 68: $188-92$.

8. Hujoel PP, Drangsholt M, Spiekerman C, et al. An exploration of the periodontitis-cancer association. Am Epidemiol 2003; 13: 312-6.

9. Michaud DS, Joshipura K, Giovannucci E, et al. A prospective study of periodontal disease and pancreatic cancer in US male health professionals. J Natl Cancer Inst 2007; 99: 171-5.

10. Michaud DS, Liu Y, Meyer M, et al. Periodontal disease, tooth loss, and cancer risk in male health professionals: a prospective cohort study. Lancet Oncol 2008; 9: $550-8$.

11. Coussens LM, Werb Z. Inflammation and cancer. Nature 2002; 420: 860-7.

12. Marx J. Cancer research. Inflammation and cancer: the link grows stronger. Science 2004; 306: 966-8.

13. Erlinger TP, Platz EA, Rifai N, et al. C-reactive protein and the risk of incident colorectal cancer. $\mathrm{J}$ Am Med Assoc 2004; 291: 585-90.

14. Heikkila $K$, Harris $R$, Lowe $G$, et al. Association of circulating $\mathrm{C}$-reactive protein and interleukin- 6 with cancer risk: findings from two prospective cohorts and a metaanalysis. Cancer Causes Control 2009; 20: 15-26.

15. Chrysanthakopoulos NA. Reasons for extraction of permanent teeth in Greece: a five-year follow-up study. Int Dent J 2011; 61: 19-24.

16. Stolzenberg-Solomon RZ, Dodd KW, Blaser MJ, et al. Tooth loss, pancreatic cancer, and Helicobacter pylori. Am J Clin Nutr 2003; 78: 176-81.

17. Rosenquist $K$, Wennerberg J, Schildt EB, et al. Oral status, oral infections and some lifestyle factors as risk factors for oral and oropharyngeal squamous cell carcinoma. A population-based case-control study in southern Sweden. Acta Otolaryngol 2005; 125: 1327-36.

18. Albandar JM. Global risk factors and risk indicators for periodontal diseases. Periodontol 2000; 29: 177-206.

19. Machtei EE, Christersson LA, Grossi SG, et al. Clinical criteria for the definition of "established periodontitis". J Periodontol 1992; 63: 206-14.

20. Machuca G, Segura-Egea JJ, Jimenez-Beato G, et al. Clinical indicators of periodontal disease in patients with coronary heart disease: A 10 years longitudinal study. Med Oral Patol Oral Cir Bucal 2012; 17: e569-74.

21. Rubin H. Fields and field cancerization: the preneoplastic origins of cancer: asymptomatic hyperplastic fields are precursors of neoplasia, and their progression to tumors can be tracked by saturation density in culture. Bio Essays 2011; 33: $224-31$.

22. Knowles J, Burgett F, Morrison E, et al. Comparison of results following three modalities of periodontal therapy related to tooth type and initial pocket depth. J Clin Periodontol 1980; 7: 32-47.

23. Wiebe CB, Putnins EE. The periodontal disease classification system of the American Academy of Periodontologyan update. J Can Dent Assoc 2000; 66: 594-7.

24. Thun MJ, Henley SJ, Burns D, et al. Lung cancer death rates in lifelong non-smokers. J Natl Cancer Inst 2006; 98: 691-9.

25. Danaei G, Vander Hoom S, Lopez AD, et al. Causes of cancer in the world: comparative risk assessment of nine behavioural and environmental risk factors. Lancet 2005; 366: 1784-93.

26. Carranza FA. Epidemiology of gingival and periodontal diseases. In: Newmann MG, Takei HH, Carranza FA, eds. Text book of Carranza's clinical periodontology. St Louis. Missouri, Saunders, 2006: 86-91.

27. Zheng W, Blot WJ, Liao ML, et al. Lung cancer and prior tuberculosis infection in Shanghai. Brit J Canc 1987; 56: $501-4$.

28. Hinds MW, Cohen HI, Kolonel LN. Tuberculosis and lung cancer risk in non smoking women. Am Rev Respir Dis 1982; 125: 776-8.

29. Astrøm AN, Rise J. Socio-economic differences in patterns of health and oral health behaviour in 25-yearold Norwegians. Clin Oral Invest 2001; 5: 122-8.

30. Thomson WM, Locker D. Dental neglect and dental health among 26-year-olds in the Dunedin Multidisciplinary Health and Development Study. Community Dent Oral Epidemiol 2000; 28: 414-8.

31. Bromen K, Pohlabeln H, Jahn I, et al. Aggregation of lung cancer in families: results from a population-based case-control study in Germany. Am J Epidemiol 2000; 152: 497-505.

32. Schwartz AG. Genetic predisposition to lung cancer. CHEST 2004; 125: 86S-9S.

33. Littman AJ, White E, Jackson LA, et al. Chlamydia pneumoniae infection and risk of lung cancer. Cancer Epidemiol Biomarkers Prev 2004; 13: 1624-30.

34. Offenbacher S. Periodontal disease: pathogenesis. Ann Periodontol 1996; 1: 821-78.

35. Tomar SL, Asma S. Smoking-attributable periodontitis in the United States: findings from NHANES III. National Health and Nutrition Examination Survey. J Periodontol 2000; 71: 743-51.

36. Bergstrom J, Eliasson S, Dock J. A 10-year prospective study of tobacco smoking and periodontal health. J Periodontol 2000; 71: 1338-47.

37. Arora M, Weuve J, Fall K, et al. An exploration of shared genetic risk factors between periodontal disease and cancers: a prospective co twin study. Am J Epidemiol 2010; 171: 253-9.

38. Hiraki A, Matsuo K, Suzuki T, et al. Teeth loss and risk of cancer at 14 common sites in Japanese. Cancer Epidemiol Biomarkers Prev 2008; 17: 1222-7. 